\title{
Strong convergence of a relaxed CQ algorithm for the split feasibility problem
}

Songnian $\mathrm{He}^{1,2^{*}}$ and Ziyi Zhao ${ }^{1}$

\section{${ }^{*}$ Correspondence:}

hesongnian2003@yahoo.com.cn

${ }^{1}$ College of Science, Civil Aviation University of China, Tianjin, 300300, China

${ }^{2}$ Tianjin Key Laboratory for Advanced Signal Processing, Civil Aviation University of China, Tianjin 300300, China

\begin{abstract}
The split feasibility problem (SFP) is finding a point in a given closed convex subset of a Hilbert space such that its image under a bounded linear operator belongs to a given closed convex subset of another Hilbert space. The most popular iterative method is Byrne's CQ algorithm. López et al. proposed a relaxed CQ algorithm for solving SFP where the two closed convex sets are both level sets of convex functions. This algorithm can be implemented easily since it computes projections onto half-spaces and has no need to know a priori the norm of the bounded linear operator. However, their algorithm has only weak convergence in the setting of infinite-dimensional Hilbert spaces. In this paper, we introduce a new relaxed CQ algorithm such that the strong convergence is guaranteed. Our result extends and improves the corresponding results of López et al. and some others.
\end{abstract}

MSC: $90 \mathrm{C} 25 ; 90 \mathrm{C} 30 ; 47 J 25$

Keywords: split feasibility problem; relaxed CQ algorithm; Hilbert space; strong convergence; bounded linear operator

\section{Introduction}

The split feasibility problem (SFP) was proposed by Censer and Elfving in [1]. It can mathematically be formulated as the problem of finding a point $x$ satisfying the property:

$$
x \in C, \quad A x \in Q,
$$

where $A$ is a given $M \times N$ real matrix (where $A^{*}$ is the transpose of $A$ ), $C$ and $Q$ are nonempty, closed and convex subsets in $\mathbb{R}^{N}$ and $\mathbb{R}^{M}$, respectively. This problem has received much attention [2] due to its applications in signal processing and image reconstruction, with particular progress in intensity-modulated radiation therapy [3-5], and many other applied fields.

We assume the SFP (1.1) is consistent, and let $S$ be the solution set, i.e.,

$$
S=\{x \in C: A x \in Q\} .
$$

It is easily seen that $S$ is closed convex. Many iterative methods can be used to solve the SFP (1.1); see [6-16]. Byrne [6, 17] was among others the first to propose the so-called CQ algorithm, which is defined as follows:

$$
x_{n+1}=P_{C}\left(x_{n}-\tau_{n} A^{*}\left(I-P_{Q}\right) A x_{n}\right),
$$

๑) 2013 He and Zhao; licensee Springer. This is an Open Access article distributed under the terms of the Creative Commons Attribution License (http://creativecommons.org/licenses/by/2.0), which permits unrestricted use, distribution, and reproduction in any medium, provided the original work is properly cited. 
where $\tau_{n} \in\left(0, \frac{2}{\|A\|^{2}}\right)$, and $P_{C}$ and $P_{Q}$ are the orthogonal projections onto the sets $C$ and $Q$, respectively. Compared with Censer and Elfving' algorithm [1], the Byrne' algorithm is easily executed since it only deal with orthogonal projections with no need to compute matrix inverses.

The CQ algorithm (1.2) can be obtained from optimization. In fact, if we introduce the (convex) objective function

$$
f(x)=\frac{1}{2}\left\|\left(I-P_{Q}\right) A x\right\|^{2},
$$

and analyze the minimization problem

$$
\min _{x \in C} f(x)
$$

then the CQ algorithm (1.2) comes immediately as a special case of the gradient projection algorithm (GPA)(For more details about the GPA, the reader is referred to [18]). Since the convex objective $f(x)$ is differentiable, and has a Lipschitz gradient, which is given by

$$
\nabla f(x)=A^{*}\left(I-P_{Q}\right) A x,
$$

the GPA for solving the minimization problem (1.4) generates a sequence $\left(x_{n}\right)$ recursively as

$$
x_{n+1}=P_{C}\left(x_{n}-\tau_{n} \nabla f\left(x_{n}\right)\right) \text {, }
$$

where the stepsize $\tau_{n}$ is chosen in the interval $\left(0, \frac{2}{L}\right)$, where $L$ is the Lipschitz constant of $\nabla f$.

Observe that in algorithms (1.2) and (1.6) mentioned above, in order to implement the $\mathrm{CQ}$ algorithm, one has to compute the operator (matrix) norm $\|A\|$, which is in general not an easy work in practice. To overcome this difficulty, some authors proposed different adaptive choices of selecting the stepsize $\tau_{n}$ (see $[6,14,19]$ ). For instance, very recently López et al. introduced a new way of selecting the stepsize [19] as follows:

$$
\tau_{n}:=\frac{\rho_{n} f\left(x_{n}\right)}{\left\|\nabla f\left(x_{n}\right)\right\|^{2}}, \quad 0<\rho_{n}<4 .
$$

The computation of a projection onto a general closed convex subset is generally difficult. To overcome this difficulty, Fukushima [20] suggested a so-called relaxed projection method to calculate the projection onto a level set of a convex function by computing a sequence of projections onto half-spaces containing the original level set. In the setting of finite-dimensional Hilbert spaces, this idea was followed by Yang [13], who introduced the relaxed CQ algorithms for solving SFP (1.1) where the closed convex subsets $C$ and $Q$ are level sets of convex functions.

Recently, for the purpose of generality, the SFP (1.1) is studied in a more general setting. For instance, Xu [12] and López et al. [19] considered the SFP (1.1) in infinite-dimensional Hilbert spaces (i.e., the finite-dimensional Euclidean spaces $\mathbb{R}^{N}$ and $\mathbb{R}^{M}$ are replaced with general Hilbert spaces). Very recently, López et al. proposed a relaxed CQ algorithm with a new adaptive way of determining the stepsize sequence $\left(\tau_{n}\right)$ for solving the SFP $(1.1)$ where the closed convex subsets $C$ and $Q$ are level sets of convex functions. This algorithm can 
be implemented easily since it computes projections onto half-spaces and has no need to know $a$ priori the norm of the bounded linear operator. However, their algorithm has only weak convergence in the setting of infinite-dimensional Hilbert spaces. In this paper, we introduce a new relaxed CQ algorithm such that the strong convergence is guaranteed in infinite-dimensional Hilbert spaces. Our result extends and improves the corresponding results of López et al. and some others.

The rest of this paper is organized as follows. Some useful lemmas are listed in Section 2. In Section 3, the strong convergence of the new relaxed CQ algorithm of this paper is proved.

\section{Preliminaries}

Throughout the rest of this paper, we denote by $H$ or $K$ a Hilbert space, $A$ is a bounded linear operator from $H$ to $K$, and by $I$ the identity operator on $H$ or $K$. If $f: H \rightarrow \mathbb{R}$ is a differentiable function, then we denote by $\nabla f$ the gradient of the function $f$. We will also use the notations:

$\rightarrow$ denotes strong convergence.

- $\rightarrow$ denotes weak convergence.

- $\omega_{w}\left(x_{n}\right)=\left\{x \mid \exists\left\{x_{n_{k}}\right\} \subset\left\{x_{n}\right\}\right.$ such that $\left.x_{n_{k}} \rightarrow x\right\}$ denotes the weak $\omega$-limit set of $\left\{x_{n}\right\}$.

Recall that a mapping $T: H \rightarrow H$ is said to be nonexpansive if

$$
\|T x-T y\| \leq\|x-y\|, \quad x, y \in H .
$$

$T: H \rightarrow H$ is said to be firmly nonexpansive if, for $x, y \in H$,

$$
\|T x-T y\|^{2} \leq\|x-y\|^{2}-\|(I-T) x-(I-T) y\|^{2} .
$$

Recall that a function $f: H \rightarrow \mathbb{R}$ is called convex if

$$
f(\lambda x+(1-\lambda) y) \leq \lambda f(x)+(1-\lambda) f(y), \quad \forall \lambda \in(0,1), \forall x, y \in H .
$$

A differentiable function $f$ is convex if and only if there holds the inequality:

$$
f(z) \geq f(x)+\langle\nabla f(x), z-x\rangle, \quad \forall z \in H .
$$

Recall that an element $g \in H$ is said to be a subgradient of $f: H \rightarrow \mathbb{R}$ at $x$ if

$$
f(z) \geq f(x)+\langle g, z-x\rangle, \quad \forall z \in H .
$$

This relation is called the subdifferentiable inequality.

A function $f: H \rightarrow \mathbb{R}$ is said to be subdifferentiable at $x$, if it has at least one subgradient at $x$. The set of subgradients of $f$ at the point $x$ is called the subdifferentiable of $f$ at $x$, and it is denoted by $\partial f(x)$. A function $f$ is called subdifferentiable, if it is subdifferentiable at all $x \in H$. If a function $f$ is differentiable and convex, then its gradient and subgradient coincide.

A function $f: H \rightarrow \mathbb{R}$ is said to be weakly lower semi-continuous (w-lsc) at $x$ if $x_{n} \rightarrow x$ implies

$$
f(x) \leq \liminf _{n \rightarrow \infty} f\left(x_{n}\right) .
$$


We know that the orthogonal projection $P_{C}$ from $H$ onto a nonempty closed convex subset $C \subset H$ is a typical example of a firmly nonexpansive mapping, which is defined by

$$
P_{C} x:=\arg \min _{y \in C}\|x-y\|^{2}, \quad x \in H .
$$

It is well known that $P_{C}$ is characterized by the inequality (for $x \in H$ )

$$
\left\langle x-P_{C} x, y-P_{C} x\right\rangle \leq 0, \quad \forall y \in C .
$$

The following lemma is not hard to prove (see $[17,21])$.

Lemma 2.1 Let $f$ be given as in (1.3). Then

(i) $f$ is convex and differential.

(ii) $\nabla f(x)=A^{*}\left(I-P_{Q}\right) A x, x \in H$.

(iii) $f$ is $w$-lsc on $H$.

(iv) $\nabla f$ is $\|A\|^{2}$-Lipschitz: $\|\nabla f(x)-\nabla f(y)\| \leq\|A\|^{2}\|x-y\|, x, y \in H$.

The following are characterizations of firmly nanexpansive mappings (see [22]).

Lemma 2.2 Let $T: H \rightarrow H$ be an operator. The following statements are equivalent.

(i) $T$ is firmly nonexpansive.

(ii) $\|T x-T y\|^{2} \leq\langle x-y, T x-T y\rangle, x, y \in H$.

(iii) $I-T$ is firmly nonexpansive.

Lemma 2.3 [23] Assume $\left(\alpha_{n}\right)$ is a sequence of nonnegative real numbers such that

$$
\alpha_{n+1} \leq\left(1-\gamma_{n}\right) \alpha_{n}+\gamma_{n} \sigma_{n}, \quad n=0,1,2, \ldots,
$$

where $\left(\gamma_{n}\right)$ is a sequence in $(0,1)$, and $\left(\sigma_{n}\right)$ is a sequence in $\mathbb{R}$ such that

(i) $\sum_{n=0}^{\infty} \gamma_{n}=\infty$.

(ii) $\lim \sup _{n \rightarrow \infty} \sigma_{n} \leq 0$, or $\sum_{n=0}^{\infty}\left|\gamma_{n} \sigma_{n}\right|<\infty$.

Then $\lim _{n \rightarrow \infty} \alpha_{n}=0$.

\section{Iterative Algorithm}

In this section, we turn to consider a new relaxed CQ algorithm in the setting of infinitedimensional Hilbert spaces for solving SFP (1.1) where the closed convex subsets $C$ and $Q$ are of the particular structure, i.e. level sets of convex functions given as follows:

$$
C=\{x \in H: c(x) \leq 0\} \text { and } Q=\{y \in K: q(y) \leq 0\} \text {, }
$$

where $c: H \rightarrow \mathbb{R}$ and $q: K \rightarrow \mathbb{R}$ are convex functions. We assume that both $c$ and $q$ are subdifferentiable on $H$ and $K$, respectively, and that $\partial c$ and $\partial q$ are bounded operators (i.e., bounded on bounded sets). By the way, we mention that every convex function defined on a finite-dimensional Hilbert space is subdifferentiable and its subdifferential operator is a bounded operator (see [24]). 
Set

$$
C_{n}=\left\{x \in H: c\left(x_{n}\right) \leq\left\langle\xi_{n}, x_{n}-x\right\rangle\right\}
$$

where $\xi_{n} \in \partial c\left(x_{n}\right)$, and

$$
Q_{n}=\left\{y \in K: q\left(A x_{n}\right) \leq\left\langle\zeta_{n}, A x_{n}-y\right\rangle\right\}
$$

where $\zeta_{n} \in \partial q\left(A x_{n}\right)$.

Obviously, $C_{n}$ and $Q_{n}$ are half-spaces and it is easy to verify that $C_{n} \supset C$ and $Q_{n} \supset Q$ hold for every $n \geq 0$ from the subdifferentiable inequality. In what follows, we define

$$
f_{n}(x)=\frac{1}{2}\left\|\left(I-P_{Q_{n}}\right) A x\right\|^{2}, \quad n \geq 0,
$$

where $Q_{n}$ is given as in (3.3). We then have

$$
\nabla f_{n}(x)=A^{*}\left(I-P_{Q_{n}}\right) A x
$$

Firstly, we recall the relaxed CQ algorithm of López et al. [19] for solving the SFP (1.1) where $C$ and $Q$ are given in (3.1) as follows.

Algorithm 3.1 Choose an arbitrary initial guess $x_{0}$. Assume $x_{n}$ has been constructed. If $\nabla f_{n}\left(x_{n}\right)=0$, then stop; otherwise, continue and construct $x_{n+1}$ via the formula

$$
x_{n+1}=P_{C_{n}}\left(x_{n}-\tau_{n} \nabla f_{n}\left(x_{n}\right)\right),
$$

where $C_{n}$ is given as (3.2), and

$$
\tau_{n}=\frac{\rho_{n} f_{n}\left(x_{n}\right)}{\left\|\nabla f_{n}\left(x_{n}\right)\right\|^{2}}, \quad 0<\rho_{n}<4 .
$$

López et al. proved that under some certain conditions the sequence $\left(x_{n}\right)$ generated by Algorithm 3.1 converges weakly to a solution of the SFP (1.1). Since the projections onto half-spaces $C_{n}$ and $Q_{n}$ have closed forms and $\tau_{n}$ is obtained adaptively via the formula (3.4) (no need to know a priori the norm of operator $A$ ), the above relaxed CQ algorithm 3.1 is implementable. But the weak convergence is its a weakness. To overcome this weakness, inspired by Algorithm 3.1, we will introduce a new relaxed CQ algorithm for solving the SFP (1.1) where $C$ and $Q$ are given in (3.1) so that the strong convergence is guaranteed.

It is well known that Halpern's algorithm has a strong convergence for finding a fixed point of a nonexpansive mapping $[25,26]$. Then we are in a position to give our algorithm. The algorithm given below is referred to as a Halpern-type algorithm [27].

Algorithm 3.2 Let $u \in H$, and start an initial guess $x_{0} \in H$ arbitrarily. Assume that the $n$th iterate $x_{n}$ has been constructed. If $\nabla f_{n}\left(x_{n}\right)=0$, then stop ( $x_{n}$ is a approximate solution of SFP (1.1)). Otherwise, continue and calculate the $(n+1)$ th iterate $x_{n+1}$ via the formula:

$$
x_{n+1}=P_{C_{n}}\left[\alpha_{n} u+\left(1-\alpha_{n}\right)\left(x_{n}-\tau_{n} \nabla f_{n}\left(x_{n}\right)\right)\right] \text {, }
$$

where the sequence $\left(\alpha_{n}\right) \subset(0,1)$ and $\left(\tau_{n}\right)$ and $\left(\rho_{n}\right)$ are given as in (3.4). 
The convergence result of Algorithm 3.2 is stated in the next theorem.

Theorem 3.3 Assume that $\left(\alpha_{n}\right)$ and $\left(\rho_{n}\right)$ satisfy the assumptions:

(a1) $\lim _{n \rightarrow \infty} \alpha_{n}=0$ and $\sum_{n=0}^{\infty} \alpha_{n}=\infty$.

(a2) $\inf _{n} \rho_{n}\left(4-\rho_{n}\right)>0$.

Then the sequence $\left(x_{n}\right)$ generated by Algorithm 3.2 converges in norm to $P_{S} u$.

Proof We may assume that the sequence $\left(x_{n}\right)$ is infinite, that is, Algorithm 3.2 does not terminate in a finite number of iterations. Thus $\nabla f_{n}\left(x_{n}\right) \neq 0$ for all $n \geq 0$. Recall that $S$ is the solution set of the SFP (1.1),

$$
S=\{x \in C: A x \in Q\}
$$

In the consistent case of the SFP (1.1), $S$ is nonempty, closed and convex. Thus, the metric projection $P_{S}$ is well-defined. We set $z=P_{S} u$. Since $z \in S \subset C_{n}$ and the projection operator $P_{C_{n}}$ is nonexpansive, we obtain

$$
\begin{aligned}
\left\|x_{n+1}-z\right\|^{2} & =\left\|P_{C_{n}}\left[\alpha_{n} u+\left(1-\alpha_{n}\right)\left(x_{n}-\tau_{n} \nabla f_{n}\left(x_{n}\right)\right)\right]-z\right\|^{2} \\
& \leq\left\|\alpha_{n}(u-z)+\left(1-\alpha_{n}\right)\left(x_{n}-\tau_{n} \nabla f_{n}\left(x_{n}\right)-z\right)\right\|^{2} \\
& \leq\left(1-\alpha_{n}\right)\left\|x_{n}-\tau_{n} \nabla f_{n}\left(x_{n}\right)-z\right\|^{2}+2 \alpha_{n}\left\langle u-z, x_{n+1}-z\right\rangle .
\end{aligned}
$$

Note that $I-P_{Q_{n}}$ is firmly nonexpansive and $\nabla f_{n}(z)=0$, it is deduced from Lemma 2.2 that

$$
\begin{aligned}
\left\langle\nabla f_{n}\left(x_{n}\right), x_{n}-z\right\rangle & =\left\langle\left(I-P_{Q_{n}}\right) A x_{n}, A x_{n}-A z\right\rangle \\
& \geq\left\|\left(I-P_{Q_{n}}\right) A x_{n}\right\|^{2} \\
& =2 f_{n}\left(x_{n}\right),
\end{aligned}
$$

which implies that

$$
\begin{aligned}
\left\|x_{n}-\tau_{n} \nabla f_{n}\left(x_{n}\right)-z\right\|^{2} & =\left\|x_{n}-z\right\|^{2}+\left\|\tau_{n} \nabla f_{n}\left(x_{n}\right)\right\|^{2}-2 \tau_{n}\left\langle\nabla f_{n}\left(x_{n}\right), x_{n}-z\right\rangle \\
& \leq\left\|x_{n}-z\right\|^{2}+\tau_{n}^{2}\left\|\nabla f_{n}\left(x_{n}\right)\right\|^{2}-4 \tau_{n} f_{n}\left(x_{n}\right) \\
& \leq\left\|x_{n}-z\right\|^{2}-\rho_{n}\left(4-\rho_{n}\right) \frac{f_{n}^{2}\left(x_{n}\right)}{\left\|\nabla f_{n}\left(x_{n}\right)\right\|^{2}} .
\end{aligned}
$$

Thus, we have

$$
\begin{aligned}
\left\|x_{n+1}-z\right\|^{2} \leq & \left(1-\alpha_{n}\right)\left\|x_{n}-\tau_{n} \nabla f_{n}\left(x_{n}\right)-z\right\|^{2}+2 \alpha_{n}\left\langle u-z, x_{n+1}-z\right\rangle \\
\leq & \left(1-\alpha_{n}\right)\left\|x_{n}-z\right\|^{2}+2 \alpha_{n}\left\langle u-z, x_{n+1}-z\right\rangle \\
& -\left(1-\alpha_{n}\right) \rho_{n}\left(4-\rho_{n}\right) \frac{f_{n}^{2}\left(x_{n}\right)}{\left\|\nabla f_{n}\left(x_{n}\right)\right\|^{2}} .
\end{aligned}
$$

Now we prove $\left(x_{n}\right)$ is bounded. Indeed, we have from (3.6) that

$$
\begin{aligned}
\left\|x_{n+1}-z\right\|^{2} & \leq\left(1-\alpha_{n}\right)\left\|x_{n}-z\right\|^{2}+2 \alpha_{n}\left\langle u-z, x_{n+1}-z\right\rangle \\
& \leq\left(1-\alpha_{n}\right)\left\|x_{n}-z\right\|^{2}+\frac{1}{4} \alpha_{n}\left\|x_{n+1}-z\right\|^{2}+4 \alpha_{n}\|u-z\|^{2},
\end{aligned}
$$


and consequently

$$
\left\|x_{n+1}-z\right\|^{2} \leq \frac{1-\alpha_{n}}{1-\frac{1}{4} \alpha_{n}}\left\|x_{n}-z\right\|^{2}+\frac{\frac{3}{4} \alpha_{n}}{1-\frac{1}{4} \alpha_{n}} \frac{16}{3}\|u-z\|^{2} .
$$

It turns out that

$$
\left\|x_{n+1}-z\right\| \leq \max \left\{\left\|x_{n}-z\right\|, \frac{16}{3}\|u-z\|\right\}
$$

and inductively

$$
\left\|x_{n}-z\right\| \leq \max \left\{\left\|x_{0}-z\right\|, \frac{16}{3}\|u-z\|\right\}
$$

and this means that $\left(x_{n}\right)$ is bounded. Since $\alpha_{n} \rightarrow 0$, with no loss of generality, we may assume that there is $\sigma>0$ so that $\rho_{n}\left(4-\rho_{n}\right)\left(1-\alpha_{n}\right) \geq \sigma$ for all $n$. Setting $s_{n}=\left\|x_{n}-z\right\|^{2}$, from the last inequality of (3.6), we get the following inequality:

$$
s_{n+1}-s_{n}+\alpha_{n} s_{n}+\frac{\sigma f_{n}^{2}\left(x_{n}\right)}{\left\|\nabla f_{n}\left(x_{n}\right)\right\|^{2}} \leq 2 \alpha_{n}\left\langle u-z, x_{n+1}-z\right\rangle
$$

Now, following an idea in [28], we prove $s_{n} \rightarrow 0$ by distinguishing two cases.

Case 1: $\left(s_{n}\right)$ is eventually decreasing (i.e. there exists $k \geq 0$ such that $s_{n}>s_{n+1}$ holds for all $n \geq k$ ). In this case, $\left(s_{n}\right)$ must be convergent, and from (3.7) it follows that

$$
\frac{\sigma f_{n}^{2}\left(x_{n}\right)}{\left\|\nabla f_{n}\left(x_{n}\right)\right\|^{2}} \leq M \alpha_{n}+\left(s_{n}-s_{n+1}\right)
$$

where $M>0$ is a constant such that $2\left\|x_{n+1}-z\right\|\|u-z\| \leq M$ for all $n \in \mathbb{N}$. Using the condition (a1), we have from (3.8) that $\frac{f_{n}^{2}\left(x_{n}\right)}{\left\|\nabla f_{n}\left(x_{n}\right)\right\|^{2}} \rightarrow 0$. Thus, to verify that $f_{n}\left(x_{n}\right) \rightarrow 0$, it suffices to show that $\left(\nabla f\left(x_{n}\right)\right)$ is bounded. In fact, it follows from Lemma 2.1 that (noting that $\nabla f_{n}(z)=0$ due to $\left.z \in S\right)$

$$
\left\|\nabla f_{n}\left(x_{n}\right)\right\|=\left\|\nabla f_{n}\left(x_{n}\right)-\nabla f_{n}(z)\right\| \leq\|A\|^{2}\left\|x_{n}-z\right\| .
$$

This implies that $\left\|\nabla f_{n}\left(x_{n}\right)\right\|$ is bounded and it yields $f_{n}\left(x_{n}\right) \rightarrow 0$, namely $\|(I-$ $\left.P_{Q_{n}}\right) A x_{n} \| \rightarrow 0$.

Since $\partial q$ is bounded on bounded sets, there exists a constant $\eta>0$ such that $\left\|\zeta_{n}\right\| \leq \eta$ for all $n \geq 0$. From (3.3) and the trivial fact that $P_{Q_{n}}\left(A x_{n}\right) \in Q_{n}$, it follows that

$$
q\left(A x_{n}\right) \leq\left\langle\zeta_{n}, A x_{n}-P_{Q_{n}}\left(A x_{n}\right)\right\rangle \leq \eta\left\|\left(I-P_{Q_{n}}\right) A x_{n}\right\| \rightarrow 0 .
$$

If $x^{*} \in \omega_{w}\left(x_{n}\right)$, and $\left(x_{n_{k}}\right)$ is a subsequence of $\left(x_{n}\right)$ such that $x_{n_{k}} \rightarrow x^{*}$, then the $w$-lsc of $q$ and (3.9) imply that

$$
q\left(A x^{*}\right) \leq \liminf _{k \rightarrow \infty} q\left(A x_{n_{k}}\right) \leq 0 .
$$


It turns out that $A x^{*} \in Q$. Next, we turn to prove $x^{*} \in C$. For convenience, we set $y_{n}:=$ $\alpha_{n} u+\left(1-\alpha_{n}\right)\left(x_{n}-\tau_{n} \nabla f_{n}\left(x_{n}\right)\right)$. In fact, since the $P_{C_{n}}$ is firmly nonexpansive, it concludes that

$$
\left\|P_{C_{n}} x_{n}-P_{C_{n}} z\right\|^{2} \leq\left\|x_{n}-z\right\|^{2}-\left\|\left(I-P_{C_{n}}\right) x_{n}\right\|^{2}
$$

On the other hand, we have

$$
\begin{aligned}
\left\|x_{n+1}-z\right\|^{2} & =\left\|P_{C_{n}} y_{n}-P_{C_{n}} z\right\|^{2} \\
& =\left\|P_{C_{n}} y_{n}-P_{C_{n}} x_{n}+P_{C_{n}} x_{n}-P_{C_{n}} z\right\|^{2} \\
& \leq\left\|P_{C_{n}} x_{n}-P_{C_{n}} z\right\|^{2}+2\left\langle P_{C_{n}} y_{n}-P_{C_{n}} x_{n}, x_{n+1}-z\right\rangle,
\end{aligned}
$$

and

$$
\begin{aligned}
\left\|P_{C_{n}} y_{n}-P_{C_{n}} x_{n}\right\| & \leq\left\|y_{n}-x_{n}\right\| \\
& =\left\|\alpha_{n} u+\left(1-\alpha_{n}\right)\left(x_{n}-\tau_{n} \nabla f_{n}\left(x_{n}\right)\right)-x_{n}\right\| \\
& \leq \alpha_{n}\left\|u-x_{n}\right\|+\tau_{n}\left\|\nabla f_{n}\left(x_{n}\right)\right\| .
\end{aligned}
$$

Noting that $\left(x_{n}\right)$ is bounded, we have from (3.10)-(3.12) that

$$
\begin{aligned}
\left\|x_{n+1}-z\right\|^{2} & \leq\left\|x_{n}-z\right\|^{2}-\left\|\left(I-P_{C_{n}}\right) x_{n}\right\|^{2}+2\left(\alpha_{n}\left\|u-x_{n}\right\|+\tau_{n}\left\|\nabla f_{n}\left(x_{n}\right)\right\|\right)\left\|x_{n+1}-z\right\| \\
& \leq\left\|x_{n}-z\right\|^{2}-\left\|\left(I-P_{C_{n}}\right) x_{n}\right\|^{2}+\left(\alpha_{n}+\frac{f_{n}\left(x_{n}\right)}{\left\|\nabla f_{n}\left(x_{n}\right)\right\|}\right) M
\end{aligned}
$$

where $M$ is some positive constant. Clearly, from (3.13), it turns out that

$$
\left\|\left(I-P_{C_{n}}\right) x_{n}\right\|^{2} \leq s_{n}-s_{n+1}+\left(\alpha_{n}+\frac{f_{n}\left(x_{n}\right)}{\left\|\nabla f_{n}\left(x_{n}\right)\right\|}\right) M .
$$

Thus, we assert that $\left\|\left(I-P_{C_{n}}\right) x_{n}\right\| \rightarrow 0$ due to the fact that $s_{n}-s_{n+1}+\left(\alpha_{n}+\frac{f_{n}\left(x_{n}\right)}{\left\|\nabla f_{n}\left(x_{n}\right)\right\|}\right) M \rightarrow 0$. Moreover, by the definition of $C_{n}$, we obtain

$$
c\left(x_{n}\right) \leq\left\langle\xi_{n}, x_{n}-P_{C_{n}}\left(x_{n}\right)\right\rangle \leq \delta\left\|x_{n}-P_{C_{n}} x_{n}\right\| \rightarrow 0 \quad(n \rightarrow \infty),
$$

where $\delta$ is a constant such that $\left\|\xi_{n}\right\| \leq \delta$ for all $n \geq 0$. The w-lsc of $c$ then implies that

$$
c\left(x^{*}\right) \leq \liminf _{k \rightarrow \infty} c\left(x_{n_{k}}\right)=0 .
$$

Consequently, $x^{*} \in C$, and hence $\omega_{w}\left(x_{n}\right) \subset S$. Furthermore, due to (2.2), we get

$$
\limsup _{n \rightarrow \infty}\left\langle u-z, x_{n}-z\right\rangle=\max _{w \in \omega_{w}\left(x_{n}\right)}\left\langle u-P_{S} u, w-P_{S} u\right\rangle \leq 0 .
$$

Taking into account of (3.7), we have

$$
s_{n+1} \leq\left(1-\alpha_{n}\right) s_{n}+2 \alpha_{n}\left\langle u-z, x_{n+1}-z\right\rangle
$$

Applying Lemma 2.3 to (3.16), we obtain $s_{n} \rightarrow 0$. 
Case 2: $\left(s_{n}\right)$ is not eventually decreasing, that is, we can find an integer $n_{0}$ such that $s_{n_{0}} \leq s_{n_{0}+1}$. Now we define

$$
V_{n}:=\left\{n_{0} \leq k \leq n: s_{k} \leq s_{k+1}\right\}, \quad n>n_{0}
$$

It is easy to see that $V_{n}$ is nonempty and satisfies $V_{n} \subseteq V_{n+1}$. Let

$$
\psi(n):=\max V_{n}, \quad n>n_{0} .
$$

It is clear that $\psi(n) \rightarrow \infty$ as $n \rightarrow \infty$ (otherwise, $\left(s_{n}\right)$ is eventually decreasing). It is also clear that $s_{\psi(n)} \leq s_{\psi(n)+1}$ for all $n>n_{0}$. Moreover,

$$
s_{n} \leq s_{\psi(n)+1}, \quad n>n_{0} .
$$

In fact, if $\psi(n)=n$, then the inequity (3.17) is trivial; if $\psi(n)<n$, from the definition of $\psi(n)$, there exists some $i \in \mathbb{N}$ such that $\psi(n)+i=n$, we deduce that

$$
s_{\psi(n)+1}>s_{\psi(n)+2}>\cdots>s_{\psi(n)+i}=s_{n}
$$

and the inequity (3.17) holds again. Since $s_{\psi(n)} \leq s_{\psi(n)+1}$ for all $n>n_{0}$, it follows from (3.8) that

$$
\frac{\sigma f_{\psi(n)}^{2}\left(x_{\psi(n)}\right)}{\left\|\nabla f_{\psi(n)}\left(x_{\psi(n)}\right)\right\|^{2}} \leq M \alpha_{\psi(n)} \rightarrow 0
$$

so that $f_{\psi(n)}\left(x_{\psi(n)}\right) \rightarrow 0$ as $n \rightarrow \infty$ (noting that $\left\|\nabla f_{\psi(n)}\left(x_{\psi(n)}\right)\right\|$ is bounded). By the same argument to the proof in case 1, we have $\omega_{w}\left(x_{\psi(n)}\right) \subset S$. On the other hand, noting $s_{\psi(n)} \leq$ $s_{\psi(n)+1}$ again, we have from (3.12) and (3.14) that

$$
\begin{aligned}
\left\|x_{\psi(n)}-x_{\psi(n)+1}\right\| \leq & \left\|x_{\psi(n)}-P_{C_{\psi(n)}} x_{\psi(n)}\right\|+\left\|P_{C_{\psi(n)}} x_{\psi(n)}-P_{C_{\psi(n)}} y_{\psi(n)}\right\| \\
\leq & \sqrt{\alpha_{\psi(n)}+\frac{f_{\psi(n)}\left(x_{\psi(n)}\right)}{\left\|\nabla f_{\psi(n)}\left(x_{\psi(n)}\right)\right\|}} \\
& \times\left(\sqrt{\alpha_{\psi(n)}+\frac{f_{\psi(n)}\left(x_{\psi(n)}\right)}{\left\|\nabla f_{\psi(n)}\left(x_{\psi(n)}\right)\right\|}}+1\right) M
\end{aligned}
$$

where $M$ is a positive constant. Letting $n \rightarrow \infty$ yields that

$$
\left\|x_{\psi(n)}-x_{\psi(n)+1}\right\| \rightarrow 0
$$

from which one can deduce that

$$
\begin{aligned}
\limsup _{n \rightarrow \infty}\left\langle u-z, x_{\psi(n)+1}-z\right\rangle & =\limsup _{n \rightarrow \infty}\left\langle u-z, x_{\psi(n)}-z\right\rangle \\
& =\max _{w \in \omega_{w}\left(x_{\psi}(n)\right)}\left\langle u-P_{S} u, w-P_{S} u\right\rangle \leq 0 .
\end{aligned}
$$

Since $s_{\psi(n)} \leq s_{\psi(n)+1}$, it follows from (3.7) that

$$
s_{\psi}(n) \leq 2\left\langle u-z, x_{\psi(n)+1}-z\right\rangle, \quad n>n_{0} .
$$


Combining (3.19) and (3.20) yields

$$
\limsup _{n \rightarrow \infty} s_{\psi(n)} \leq 0
$$

and hence $s_{\psi(n)} \rightarrow 0$, which together with (3.18) implies that

$$
\begin{aligned}
\sqrt{s_{\psi(n)+1}} & \leq\left\|\left(x_{\psi(n)}-z\right)+\left(x_{\psi(n)+1}-x_{\psi(n)}\right)\right\| \\
& \leq \sqrt{s_{\psi(n)}}+\left\|x_{\psi(n)+1}-x_{\psi(n)}\right\| \rightarrow 0,
\end{aligned}
$$

which, together with (3.17), in turn implies that $s_{n} \rightarrow 0$, that is, $x_{n} \rightarrow z$.

Remark 3.4 Since $u$ can be chosen in $H$ arbitrarily, one can compute the minimum-norm solution of SFP (1.1) where $C$ and $Q$ are given in (3.1) by taking $u=0$ in Algorithm 3.2 whether $0 \in C$ or $0 \notin C$.

\section{Competing interests}

The authors declare that they have no competing interests.

\section{Authors' contributions}

All the authors read and approved the final manuscript.

\section{Acknowledgements}

The authors wish to thank the referees for their helpful comments, which notably improved the presentation of this manuscript. This work was supported by the Fundamental Research Funds for the Central Universities (ZXH2012K001) and in part by the Foundation of Tianjin Key Lab for Advanced Signal Processing.

\section{Received: 15 November 2012 Accepted: 9 April 2013 Published: 22 April 2013}

\section{References}

1. Censor, $Y$, Elfving, T: A multiprojection algorithm using Bregman projection in product space. Numer. Algorithms 8(2-4), 221-239 (1994)

2. López, G, Martín-Márquez, V, Xu, HK: Iterative algorithms for the multipul-sets split feasiblity problem. In: Censor, Y, Jiang, M, Wang, G (eds.) Biomedical Mathematics Promising Directions in Imaging, Therapy Planning and Inverse Problems, pp. 243-279. Medical Physics Publishing, Madison, WI (2010).

3. Censor, Y, Bortfeld, T, Martin, B, Trofimov, A: A unified approach for inversion problems in intensity-modulated radiation therapy. Phys. Med. Biol. 51, 2353-2365 (2003)

4. Censor, Y, Elfving, T, Kopf, N, Bortfeld, T: The multiple-sets split feasibility problem and its applications for inverse problem. Inverse Probl. 21, 2071-2084 (2005)

5. López, G, Martín-Márquez, V, Xu, HK: Perturbation techniques for nonexpansive mappings with applications. Nonlinear Anal., Real World Appl. 10, 2369-2383 (2009)

6. Byrne, C: Iterative oblique projection onto convex sets and the split feasibity problem. Inverse Probl. 18, 441-453 (2002)

7. Dang, Y, Gao, Y: The strong convergence of a KM-CQ-like algorithm for a split feasibility problem. Inverse Probl. 27, 015007 (2011)

8. Qu, B, Xiu, N: A note on the CQ algorithm for the split feasibility problem. Inverse Probl. 21, 1655-1665 (2005)

9. Schöpfer, F, Schuster, T, Louis, AK: An iterative regularization method for the solution of the split feasibility problem in Banach spaces. Inverse Probl. 24, 055008 (2008)

10. Wang, $F, X u, H K$ : Approximating curve and strong convergence of the $C Q$ algorithm for the split feasibility problem. J. Inequal. Appl. 2010, 102085 (2010)

11. Xu, HK: A variable Krasonosel'skii-Mann algorithm and the multiple-set split feasibility problem. Inverse Probl. 22, 2021-2034 (2006)

12. $\mathrm{Xu}, \mathrm{HK}$ : Iterative methods for the split feasibility problem in infinite-dimensional Hilbert spaces. Inverse Probl. 26, $105018(2010)$

13. Yang, Q: The relaxed CQ algorithm for solving the split feasibility problem. Inverse Probl. 20, 1261-1266 (2004)

14. Yang, Q: On variable-set relaxed projection algorithm for variational inequalities. J. Math. Anal. Appl. 302, 166-179 (2005)

15. Zhao, J, Yang, Q: Generalized KM theorems and their applications. Inverse Probl. 22(3), 833-844 (2006)

16. Zhao, J, Yang, Q: Self-adaptive projection methods for the multiple-sets split feasibility problem. Inverse Probl. 27, 035009 (2011)

17. Byrne, C: A unified treatment of some iterative algorithms in signal processing and image reconstruction. Inverse Probl. 20(1), 103-120 (2004) 
18. Figueiredo, MA, Nowak, RD, Wright, SJ: Gradient projection for space reconstruction: application to compressed sensing and other inverse problems. IEEE J. Sel. Top. Signal Process. 1, 586-598 (2007)

19. López, G, Martín-Márquez, V, Wang, FH, Xu, HK: Solving the split feasibility problem without prior knowledge of matrix norms. Inverse Probl. (2012). doi:10.1088/0266-5611/28/8/085004

20. Fukushima, M: A relaxed projection method for variational inequalities. Math. Program. 35, 58-70 (1986)

21. Aubin, JP: Optima and Equilibria: An Introduction to Nonlinear Analysis. Springer, Berlin (1993)

22. Goebel, K, Kirk, WA: Topics on Metric Fixed Point Theory. Cambridge University Press, Cambridge (1990)

23. Xu, HK: Iterative algorithms for nonlinnear operators. J. Lond. Math. Soc. 66, 240-256 (2002)

24. Bauschke, HH, Borwein, JM: On projection algorithms for solving convex feasibility problem. SIAM Rev. 38, 367-426 (1996)

25. Suzuki, T: A sufficient and necessary condition for Halpern-type strong convergence to fixed points of nonexpansive mappings. Proc. Am. Math. Soc. 135, 99-106 (2007)

26. Xu, HK: Viscosity approximation methods for nonexpansive mappings. J. Math. Anal. Appl. 298, $279-291$ (2004)

27. Halpern, B: Fixed points of nonexpanding maps. Bull. Am. Math. Soc. 73, 957-961 (1967)

28. Maingé, PE: A hybrid extragradient-viscosity method for monotone operators and fixed point problems. SIAM J. Control Optim. 47, 1499-1515 (2008)

doi:10.1186/1029-242X-2013-197

Cite this article as: He and Zhao: Strong convergence of a relaxed CQ algorithm for the split feasibility problem. Journal of Inequalities and Applications 2013 2013:197.

\section{Submit your manuscript to a SpringerOpen ${ }^{\circ}$ journal and benefit from:}

- Convenient online submission

- Rigorous peer review

- Immediate publication on acceptance

- Open access: articles freely available online

- High visibility within the field

- Retaining the copyright to your article 